\title{
Estado, planificación y cambio institucional en Venezuela $(1936-1941)^{*}$
}

\section{State, planning and institutional change in Venezuelan (1936-1941)}

Luz Marina Rondón de Delgado** Virginia Rondón de Medina****

Recibido: 17 de Octubre de 2012

Aprobado: 7 de Marzo de 2013

\section{Resumen}

En el presente trabajo se muestra que el Sistema Nacional de Planificación de 1958 fue el resultado de un proceso que se inició con el ascenso al poder de Eleazar López Contreras en 1936. Así, durante su gobierno, concurrieron acciones ejecutadas por el Estado que teóricamente han sido definidas como facilitadoras de los objetivos de la

* Cómo citar este artículo: Rondón, L. \& Rondón, V. (2013). Estado, planificación y cambio institucional en Venezuela (1936-1941). Revista CIFE, 15 (23), pp. 49-76.

** Profesora e investigadora en el Instituto de Invstigaciones Económicas y Sociales, Facultad de Ciencias Económicas y Sociales, Universidad de los Andes, Mérida, Venezuela. Correo electrónico: uzmarrq@ula.ve.

*** Profesora e investigadora en el Departamento de Economía, Facultad de Ciencias Económicas y Sociales, Universidad de los Andes, Mérida, Venezuela. Correo electrónico: virginia.rondon@gmail.com. 
ISSN: 0124-3551 / Año 15, No 23 / julio-diciembre / pp. 49-76

planificación: Un diagnóstico y un conjunto de políticas presentados en el Programa de Febrero de 1936, y un plan de acción con su respectivo presupuesto, propuesto en el Plan Trienal de 1939. La contundencia de la intervención se plasmó en las distintas realizaciones, tantos institucionales como materiales, que llevaron a Venezuela a la modernización. El estudio se desarrolla siguiendo el enfoque neoinstitucional de Douglass North.

Palabras clave: Neoinstitucionalismo, planificación, intervencionismo, Programa de Febrero, Plan Trienal.

Clasificación JEL: H50, H83, N46, O54

\begin{abstract}
This paper shows that the National Planning System was the result of a process that began with the rise to power of Eleazar Lopez Contreras in 1936. During his government concurred actions taken by the State that have been defined theoretically as facilitators of the planning objectives: diagnosis and policies presented in the February Program of 1936 and an action plan with its budget (Triennial Plan) proposed in 1939. The strength of the intervention was reflected in the various embodiments as many institutional materials took Venezuela to modernization. The study developed following the neoinstitutional approach of Douglass North.
\end{abstract}

Keywords: Neo-institucionalism, planning, interventionism, February Program, Triennial Plan

Classification JEL: H50, H83, N46, O54 


\section{Introducción}

La planificación económica, entendida como una disciplina decisiva para racionalizar la toma de decisiones públicas para el desarrollo, surgió en América Latina como un método para abordar la urgencia de la industrialización en la década del cincuenta y se consolidó en los años sesenta. Los cambios estructurales inherentes al proceso de transformación de economías tradicionales a economías industrializadas exigían racionalidad y visión en la política pública, además de importantes inversiones en infraestructura. Por ello, la planificación se constituyó como el mecanismo necesario para lograr las transformaciones que demandaba la industrialización y de este modo acelerar el crecimiento económico.

El concepto de planificación que se impulsó en América Latina tuvo su origen en una propuesta sistematizada por la Comisión Económica para América Latina (CEPAL, creada en 1948), más tarde adoptada en una reunión intergubernamental realizada en Punta del Este en el año 1961, cuando se acordó realizar en el continente La Alianza para el Progreso ${ }^{1}$. En consecuencia, comenzaron a aparecer los organismos centrales de planificación que fueron encargados de orientar, coordinar y apoyar las acciones del Estado para alcanzar la industrialización mediante el modelo de sustitución de importaciones.

En Venezuela, el sistema de planificación concebido por la CEPAL se adoptó en 1958 mediante el "Decreto 492" (Junta de Gobierno de la República de Venezuela, 1958), que ordenó la creación de un Sistema de Planificación. Desde ese momento se asumió a la planificación no solo como una propuesta técnica para formular planes, sino como un mecanismo de acción racional para el conocimiento y la toma de decisiones en materia económica y social. La institucionalización de la planificación en Venezuela, y en la mayoría de los países de América Latina, fue el resultado de un proceso de reflexión y conocimiento de la realidad particular de las economías de la región, proceso que tuvo sus orígenes en la década del veinte en países como Argentina, Brasil y Chile y, en los años treinta para Venezuela, Colombia, México, entre otros.

Ahora bien, si se considera la planificación como el "método de intervención para producir un cambio en el curso tendencial de los eventos" (Lira, 2006, p. 7), en Venezuela la planificación comenzó en 1936 con el diagnóstico y las políticas plasmadas en el

1 En la conferencia de Punta del Este (Uruguay), que se realizó del 5 al 17 de agosto de 1961, se aprobó la creación de la Alianza para el Progreso (ALPRO). En el texto oficial de su Constitución se estableció su objetivo general: "mejorar la vida de todos los habitantes del continente"; para ello se proclamaron varias medidas de carácter social (educación, sanidad, vivienda...), político (defendiendo la formación de sistemas democráticos, según el principio de autodeterminación de los pueblos) y económico (limitación de la inflación, mejora de la balanza de pagos, siempre bajo la iniciativa privada). Para garantizar estos objetivos, Estados Unidos se comprometió a cooperar en aspectos técnicos y financieros. La opinión pública recibió con entusiasmo esta declaración, pero el programa fracasó debido a que, tras el asesinato de Kennedy, sus sucesores limitaron la ayuda financiera estadounidense en América Latina (Alianza para el Progreso, s. f. Documentos básicos. Punta del Este, Uruguay). 
documento conocido como Programa de Febrero del General Eleazar López Contreras, presidente constitucional de Venezuela (1936-1941). El programa sintetizó la acción del Estado para la conducción de las fuerzas sociales y económicas del país con el propósito de lograr su modernización.

La percepción subjetiva de López Contreras sobre la realidad del país y su visión de modernidad produjeron un nuevo marco institucional (North, 1993; 2007): [1] un nuevo orden legal (normas formales), [2] nuevas limitaciones informales y [3] nuevas estrategias para imponer los cambios en las condiciones de atraso (gomecistas). Estos cambios marcaron la vía para las subsecuentes transformaciones en las reglas de juego venezolanas durante los años y décadas siguientes para mejorar el desempeño económico.

Es conveniente advertir que para 1936 no existían técnicas de programación general. Estas aparecieron en la década del cuarenta y se desarrollaron en la década del cincuenta. Su finalidad era "llegar a un cuadro general o a una serie de cifras que sirvieran de armazón al desarrollo posible de una economía" (Tinberger, 1959, p. 15). Sin embargo, en el gobierno de López Contreras, la ausencia de un método sistematizado no impidió la acción racional y coordinada de los actores políticos para inducir cambios en la estructura institucional de Venezuela con el objeto de modificar el curso tendencial en las distintas áreas y, con ello, marcar una nueva vía para el desarrollo capitalista posterior. En este orden de ideas, en el presente trabajo se sostiene que para llegar a la creación del Sistema Nacional de Planificación de 1958, se recorrió un camino que se inició en 1936 con el Programa de Febrero y el Plan Trienal de 1938. Estos hechos marcaron la dependencia de la vía (path dependency, North, 1993) para la institucionalización posterior de la planificación en Venezuela. De esta manera se destaca que aunque el intervencionismo en el país ha existido a lo largo de su historia republicana, serán las acciones emprendidas en el gobierno de López Contreras para lograr la modernización, los primeros referentes de la planificación en Venezuela que introdujeron el cambio institucional en la acción del Estado.

\section{Consideraciones sobre la planificación en América Latina}

En las décadas que siguieron a la Segunda Guerra Mundial, la búsqueda del desarrollo económico ${ }^{2}$ se materializó en la aceptación de la planificación del desarrollo como una vía más segura y directa para alcanzarlo. Se consideraba que el sistema de planificación

2 Ilma Adelman (1961) definió desarrollo económico "como el proceso por medio del cual se transforma una economía cuyo ingreso por habitante tiene una tasa de crecimiento pequeña o negativa, en una economía en la cual el ingreso por persona tiene una tasa significativa del incremento autosostenido como una característica permanente a largo plazo. Denominaremos subdesarrolladas a una sociedad en la cual el desarrollo económico es posible pero incompleto" (p. 11). 
centralizada era el mecanismo institucional y organizativo fundamental, capaz de garantizar una elevada tasa de crecimiento económico en los países no desarrollados.

Por planificación del desarrollo o planificación económica se entendía el propósito deliberado por parte del Estado de coordinar a largo plazo el proceso de decisión económica. Esta coordinación comprendía la modificación, dirección y, en algunos casos, el control del nivel del PIB real per cápita y su tasa de crecimiento, con el fin de lograr un conjunto determinado de objetivos relacionados con el crecimiento económico y el bienestar social (Todaro, 1988, p. 538). El objetivo de la planificación económica consistía en sistematizar los esfuerzos públicos y privados para producir cambios económicos que produjeran la transformación de las economías, particularmente las economías subdesarrolladas, evitando los errores que hasta ese momento habían sucedido en los países desarrollados en los cuales "el progreso no había seguido un ritmo regular, sino que se interrumpía con trastornos y crisis” (Tinberger, 1959, p. 7).

En este sentido, la principal tarea de la planificación en los países subdesarrollados era la de "movilizar los recursos limitados de forma que [dieran] lugar al cambio estructural necesario para lograr un crecimiento sostenido y equilibrado de toda la economía" (Todaro, 1988, p. 539) que ameritaba la reflexión y análisis de las condiciones particulares de cada país. Primeramente, la política económica atendería las condiciones macroeconómicas generales como la elevada inflación, el desempleo significativo y el desequilibrio en la balanza de pagos, para luego dedicarse a diseñar e implantar políticas en gran escala que desarrollarían el producto nacional mediante la producción del sector industrial, el aumento de las exportaciones, el consumo y la inversión.

En América Latina, la planificación como mecanismo racional que se proponía explorar el futuro mediante una metodología en proyecciones de largo plazo "apareció teóricamente en la década de los años cuarenta" (Matus, 1981, p. 7), como herramienta para resolver los problemas estructurales que caracterizaban la región. En contraste, el tipo de planificación que se utilizó en Europa consistió en técnicas de programación a mediano y corto plazo, para atender las circunstancias históricas de la reconstrucción de los países devastados por las guerras mundiales y la implantación del sistema socialista en la Unión de Repúblicas Soviéticas Socialistas (URSS). No obstante, las técnicas europeas, aunque con plazos más inmediatos, sirvieron para evidenciar la utilidad de los planes y la importancia de racionalizar la toma de decisiones públicas.

Por otra parte, el establecimiento de la planificación en América Latina surgió como "un proceso previo de formación de conciencia para demostrar su necesidad" (Matus, 1981, p. 8). Esto quiere decir, siguiendo las ideas de North (2007), que la adopción de la planificación en América Latina, supuso primeramente la comprensión de la realidad de la región por "los emprendedores políticos y económicos", quienes elaboraron un conjunto 
de políticas partiendo de un "sistema de creencias"3 relacionadas con la pertinencia de la planificación como mecanismo para afectar el desempeño económico y social.

En efecto, desde la década de los años veinte ya figuraban los intelectuales que introdujeron las reflexiones y los análisis que necesitaba la región para comprender su realidad y, con ello, conocer los obstáculos y dificultades para generar los cambios estructurales que exigía la industrialización. Los aportes estuvieron encabezados por Raúl Prebisch, entre un significativo grupo de intelectuales que produjeron una teoría del desarrollo para América Latina4.

El proceso de industrialización en América Latina se había iniciado desde la década de los años veinte en países como Argentina, Brasil y Chile (Bénard, 1965, p. 34), y se constituyó en el modo de elevar la productividad de la fuerza de trabajo y con ello mejorar el ingreso y el desempeño económico. Sin embargo, la industrialización no había alcanzado a la mayoría de los países de la región, y ameritó preparar los cambios en las condiciones que hasta la década del cincuenta no se habían materializado. En la consecución de este objetivo, la propuesta de la CEPAL promovía la industrialización mediante la sustitución de importaciones 5 .

La baja productividad de una gran parte de la fuerza de trabajo, como rasgo fundamental de las economías de América Latina, exigió la elaboración de planes nacionales para la promoción de la industrialización. Estos planes contenían diagnósticos y proyecciones globales; los primeros para identificar los obstáculos y las segundas para captar los efectos de su persistencia o programar oportunamente su eliminación. De este modo, fue posible establecer los fines que se pretendían alcanzar mediante el sistema de proyecciones que recibió el nombre de "plan" (Matus, 1981, p. 10).

3 North (2007, p. 19) sostuvo que "nadie conoce nunca la 'realidad de un sistema político y económico, pero es un hecho que los seres humanos construyen elaboradas creencias acerca de la naturaleza de esa 'realidad', creencias que son a la vez un modelo positivo del funcionamiento concreto del sistema y un modelo normativo de su funcionamiento ideal. Dentro de una sociedad, el sistema de creencias puede ser objeto de una amplia aceptación, como reflejo de un consenso en esa materia, o bien puede haber creencias muy dispares, reflejando divisiones fundamentales en la percepción de la sociedad. Las creencias dominantes -las de los emprendedores políticos y económicos en condiciones de elaborar políticas- desembocan, con el paso del tiempo, en el despliegue de una complicada estructura de instituciones que determinan el desempeño económico y político.

4 Parra Luzardo (2006, p. 14) señaló como miembros de los pensadores latinoamericanos que contribuyeron a formar la conciencia sobre la necesidad de reflexionar acerca de las características particulares de América Latina a Raúl Prebisch, Alonso Aguilar, Fernando Carmona, André Gunder Frank, José Consuegra H., Oswaldo Sunkel, Antonio García, Pedro Paz, Aníbal Pinto, Pedro Vuskovic, René Báez, Julián Sabogal Tamayo, Isidro Parra Paz, Theotonio Dos Santos, Aldo Ferrer, Sergio Bagú, Pablo González Casanova, Jesús Silva Herzog, Ruy Marini, Marcos Kaplan, Aníbal Quijano, Aníbal Pinto, Eduardo Galeano, Josué de Castro, entre otros.

5 Los instrumentos aplicados durante el proceso de substitución de importaciones eran, entre otros, los aranceles protectores y/o el control de cambio; preferencias especiales para las nuevas ramas industriales y para las empresas nacionales y extranjeras importadoras de bienes de producción; cambios preferenciales en cuanto a la importación de materias primas industriales y semiprocesadas; facilidades de crédito por parte de los bancos nacionales de desarrollo para selectas ramas industriales; estímulo estatal al desarrollo de infraestructuras que propiciaran el desarrollo industrial; participación directa del estado en algunas ramas de la industria, especialmente de la industria pesada (Borbély, 1991, p. 27). 
El plan debía orientarse a diseñar un conjunto de políticas que tomaran en cuenta los aspectos sugeridos por Prebisch (1983): i) evitar la asignación de recursos productivos adicionales a las actividades de importación de bienes primarios y desviarlos hacia la producción industrial; ii) elevar la productividad de toda la fuerza de trabajo mediante el avance del progreso tecnológico; iii) cambiar la estructura de la producción que se correspondería con una elevada elasticidad de la demanda de manufacturas (p. 1.079).

Por su parte, Tinbergen (1959) señalaba que el desarrollo económico podía promoverse mediante una "política de desarrollo", que contara con las fuerzas poderosas de la iniciativa privada, pero con una acción del Estado mucho más contundente. Para ello, este autor señalaba cuatro aspectos esenciales de la política de desarrollo, a saber:

a) crear condiciones generales favorables al desarrollo; b) familiarizar al gobierno mismo, a la comunidad mercantily al público en general con las potencialidades yventajas del desarrollo; c) promover (y ejecutar) una serie de inversiones, por lo general del tipo básico - programa de inversión del gobierno, o el plan, consistente de una serie de proyectos públicos; y d) tomar medidas destinadas a facilitar y a estimular la actividad y las inversiones privadas p. 7).

Podría afirmarse que estas ideas de desarrollo económico y de la planificación expuestas por Prebisch y Tinberger representan apropiadamente las percepciones subjetivas de los funcionarios públicos responsables de cambiar el marco institucional en América Latina. Para ello, la intención de los actores (emprendedores políticos y económicos) de generar instituciones (políticas, sociales y económicas) constituía el fundamento del cambio.

\section{Las ideas de Alberto Adriani y Manuel Egaña sobre la intervención del Estado en la economía}

Alberto Adriani y Manuel Egaña son dos reconocidos pensadores y políticos venezolanos que ocuparon cargos ministeriales durante el gobierno del General Eleazar López Contreras. El primero como Ministro de Agricultura y Cría y Ministro de Hacienda (1936), y el segundo como Ministro de Fomento (1938-1941). La rigurosa formación académica vinculada a una profunda reflexión sobre la situación social, económica y política del país, los convirtieron en figuras sobresalientes, no solo de la época sino de la historia reciente del país.

Rivas y Maldonado (2010) afirmaron que el padre de la planificación en Venezuela fue Alberto Adriani. Se comparte este planteamiento por cuanto Adriani fue el primer pensador y político venezolano que propuso la adopción de un "programa estatal", concebido como un "método" con la característica particular de ser "racional" (Adriani, 1984). Adriani entendía que el desarrollo económico del país solo se conseguiría mediante el 
diseño e implantación de un conjunto de medidas que abarcaran los distintos aspectos de la vida nacional con base en la ciencia y el uso de la tecnología.

Sin embargo, Adriani tuvo su propia curva de aprendizaje con relación al Estado, lo cual puede verificarse en el estudio que hiciera Szinetar Gabaldón (1998) sobre su obra que calificó como "Proyecto de Cambio Social". En su estudio Szinetar identificó varias formulaciones del proyecto de Adriani. En la primera etapa (identificable en textos inéditos escritos entre 1914 y 1918) Adriani no introdujo el concepto de Estado. Le otorgó al gobierno el papel de crear las condiciones necesarias para estimular la iniciativa privada, y por tanto el "gobierno no sería interventor" (Szinetar, 1998, p. 17). En la segunda etapa (construida en los escritos de 1918 a 1925, inéditos unos y publicados otros), Adriani planteó, en 1919, que el Estado latinoamericano debía convertirse en uno moderno que veía como un "Estado providencia", que Szinetar identificó con la concepción de Estado de bienestar impuesta después de la Segunda Guerra Mundial. Es decir, será a partir de esta fecha cuando Adriani otorgó al Estado un rol estelar en la consecución del bienestar social; continuó observando las experiencias de otros países y parte de sus conclusiones se basaron en la admiración por la obra del francés Charles Maurras con su antidemocratismo y antiparlamentarismo, del ministro alemán Walter Rhatenau quien, frente a la grave situación alemana, después de la Primera Guerra Mundial, propuso un nuevo "programa de vida" (Adriani, 1922, p. 87), y del fascismo italiano, el cual compartía tanto por su crítica al anarquismo y al comunismo como por los resultados obtenidos por ese régimen.

En la tercera etapa de la formulación del Proyecto de Adriani (en ensayos y documentos oficiales que presentó entre 1926 y 1936), continuó otorgándole al Estado el papel principal para la obtención del cambio. De allí su concepción sobre el "Estado totalitario" que asoció con la necesidad de que el Estado interviniese en todos los aspectos de la nación para realizar la unidad y lograr el desarrollo (Szinetar Gabaldón, 1998).

Ahora bien, Adriani plasmó en distintos trabajos sus ideas sobre cómo debería ser la actuación del Estado en distintas materias. Particularmente interesan las relacionadas con la inmigración y la colonización, el café y la agricultura en general, las comunicaciones y la política monetaria (ver Adriani, 1984b). En síntesis, consideraba que era necesario que el Estado dirigiera estas materias y creía que se debía concebir un programa que las contemplara:

Es decir, debemos adoptar una política económica [...] sería fatal abandonar a sí mismas las fuerzas económicas de un país; permitir que las energías privadas, casi siempre incapaces de percibir y renuentes en respetar el interés colectivo, lleven a cabo, sin control alguno, la

6 Miguel Szinetar Gabaldón (1998) definió Proyecto de Cambio Social como "un conjunto de ideas integradas en torno a una propuesta para cambiar la sociedad; a una representación anticipada de la acción, o de las acciones que tendrían que efectuarse para lograr el cambio de una sociedad determinada" (p. 3). 
explotación de los recursos naturales, las vías de comunicación y muchos otros actos económicos que afecten toda la colectividad [...].

Se trata para nosotros de formular un programa que, en sus grandes líneas, nos señale la ruta durante un largo espacio de tiempo y nos asegure las ventajas de la continuidad del esfuerzo. Tal programa no debería formularse sin previo y cabal estudio, por parte de una Comisión de técnicos idóneos de los diversos aspectos de nuestra vida económica (Adriani 1984, pp. 103-104).

En cuanto a Manuel Egaña, en pleno ejercicio de sus funciones ministeriales, justificó la acción del Estado y defendió el criterio sobre la intervención del Estado en la vida económica:

La doctrina venezolana no puede calificarse de otra manera que de intervencionista. En Venezuela nunca han sido respetados como dogmas intangibles los expresados principios del liberalismo económico, ni se le ha puesto al Estado, como límites de la acción, el conservar el orden, el administrar la justicia y el defender la independencia de la nación (Ministerio de Fomento, 1939, pp. 8-9).

Esta justificación de la acción intervencionista del Estado venezolano sirvió de antesala no solo para justificar la existencia histórica del intervencionismo, sino que fue utilizada para argumentar el papel que desempeñó el gobierno de López Contreras. De esta manera, el presidente dedicó sus esfuerzos para llevar a Venezuela a la modernidad. Gracias a los recursos petroleros, pudo emprender las reformas institucionales. De igual manera, Egaña identificó las siguientes razones que recomendaban un mínimo de intervención estatal:

[1] Conviene estimular la acción de la libre competencia para que se reduzcan los precios de los mercados concurridos, con lo cual se bajará el costo de la vida, desmedidamente elevado.

[2] Es aconsejable respetar los amplios márgenes de beneficio que puede reportar una inversión aventurada y azarosa de capital y de trabajo, por ser Venezuela país en proceso de crecimiento rápido, con abundantes riquezas naturales pero con grandes dificultades y riesgos para su explotación y consiguiente valorización.

[3] El instrumento administrativo para una intensa acción económica es todavía insuficiente.

[4] Conviene reducir el coeficiente del presupuesto nacional dedicado a retribución de funcionarios y servicios. (Ministerio de Fomento, 1939, pp. pp. 8-13).

En 1940, nuevamente Egaña, en la introducción de la Memoria de Fomento correspondiente a ese año, expresó:

Si bien es cierto que la acción del Estado debe ser estimulante, orientadora y tuitiva, no es menos cierto que esa acción debe circunscribirse al desarrollo de la Nación como un conjunto armónico. El Estado debe intervenir, bien sea de manera directa o indirecta, mediata o inmediata, para promover, alentar, fortalecer actividades económicas y para proteger intereses de la colectividad cuando sean amenazados o perjudicados. No debe pretender 
sustituir a los particulares en la posesión y administración de las empresas privadas, porque con ello malograría las mejores fuerzas del progreso humano (Memoria de Fomento, 1940, p. 7).

En las introducciones a las memorias, Egaña dejó claros sus criterios sobre la intervención económica del Estado. En primer lugar, señaló que el primer deber de este es la conservación del orden interno y la independencia del exterior. En el ámbito internacional, la Segunda Guerra Mundial estaba generando una nueva crisis de la cual Venezuela no escapaba. En segundo lugar, el ministro reforzó el razonamiento de que el Estado debía intervenir sin obstaculizar el desempeño de la actividad privada. Por último, Egaña reafirmó la responsabilidad particular del Estado en el desarrollo y progreso de la Nación y, en virtud de los recursos petroleros, invertirlos racionalmente en el fomento de la producción.

Por lo dicho en los párrafos precedentes, se puede señalar que Alberto Adriani y Manuel Egaña fueron destacados políticos que contribuyeron a la configuración de la modernización de la economía nacional. Junto con otros integrantes del gabinete de Eleazar López Contreras, generaron reflexiones e indujeron los cambios incrementales que transformaron el país.

Así, en el Programa de Febrero, que se considera la raíz del cambio institucional en esa época, se pueden identificar las ideas de ambos autores. Por ejemplo, el hecho que uno de los puntos fundamentales del plan fuese la agricultura y cría y que no se contemplara un apartado sobre los hidrocarburos, denota la influencia de Adriani. Una posible hipótesis que explicaría el motivo de esta notable omisión sería la fuerte influencia de las creencias de Adriani y su fuerte sesgo agrícola, dado que para él este era el sector privilegiado en el que se podrán identificar las oportunidades de "actividad y riqueza para el país” (1984)7. De la misma forma, la propuesta de creación del Banco Central de Emisión (López Contreras, 1966) se refiere a las ideas de Egaña. Crazut (1989) asomó la tesis de que fue Egaña quien influenció en el ánimo de López Contreras para que incluyera la creación del organismo como objetivo prioritario. Ello por cuanto esta era una idea que Egaña había sugerido en distintos escenarios.

\section{El Programa de Febrero y las nociones precursoras sobre planificación en Venezuela (1936-1941)}

A la muerte del General Juan Vicente Gómez, gobernante de Venezuela durante el periodo 1908-1935, las condiciones sociales, económicas y políticas de Venezuela ameritaban cambios fundamentales para favorecer su superación. Era necesaria una mayor intervención del Estado que efectivamente se inició con el ascenso al poder del General

7 En todo caso, para una descripción de los conflictos alrededor del cambio institucional petrolero entre 1936 y 1943 véanse los trabajos de Maldonado-Veloza (2012a y 2012b). 
Eleazar López Contreras en 1936, y la implantación de un importante conjunto de reformas institucionales.

La intervención del Estado en la economía, que ha existido a lo largo de la historia republicana, se vigorizó con el incremento de los ingresos fiscales provenientes de la actividad petrolera a partir de la segunda década del siglo veinte. Estos recursos fueron utilizados por el gobierno del General Juan Vicente Gómez para el desarrollo de obras de infraestructura, y posteriormente, por el General Eleazar López Contreras para poner en marcha un proyecto de modernización del país con base en el uso racional de los ingresos petroleros, que permitieron iniciar el proceso de transformación y sentar las bases estructurales para convertir al país agrícola en uno moderno.

En este sentido, el intervencionismo durante el gobierno de López Contreras se reconoce, entre otros, como un aspecto esencial del inicio de la planificación en Venezuela, por cuanto involucró un proceso de cambios en el sistema de creencias que reflejaban la transformación en la percepción acerca del rol del Estado y de los mecanismos para afectar la actividad económica y social. Con él se comenzó a visualizar un país con una población más alfabetizada, sana y capacitada para el trabajo; una sociedad que permitiera el desarrollo de las fuerzas productivas y el establecimiento del sistema capitalista (garantizar derechos laborales, eliminar monopolios, establecer un sistema impositivo moderno, establecer derechos de propiedad, erigir instituciones formales en materia comercial, agrícola, industrial y petrolera). En este contexto, las vías y medios de comunicación adquirieron nuevas funciones acordes a la visión de desarrollo económico, político y social del país, es decir, permitirían la colonización agrícola, el acercamiento de las distintas regiones y el incremento del comercio interno y externo.

En lo relativo a la política comercial se vislumbró la creación de la autoridad monetaria, es decir, del Banco Central de Venezuela, la adopción de políticas proteccionistas y la celebración de tratados bilaterales y multilaterales conjuntamente con el sector privado.

La agricultura y cría ocupó un lugar central en esa visión de país. Debía convertirse en un sector competitivo mediante la formación y capacitación a la población para las actividades del campo y de profesionales en el área. También se experimentaría con nuevos cultivos, se contribuiría a potenciar las actividades pecuarias y se introducirían nuevas actividades como la pesca, se haría un inventario de los recursos y de las tierras baldías, se establecerían políticas de conservación de los distintos recursos naturales y se cuidaría de la salud animal y vegetal (López Contreras, 1966).

Con respecto a la industria, en este periodo se concebía la industrialización como la condición imperante para alcanzar mayores estadios de prosperidad y de desempeño económico; por tanto, era necesario diseñar un conjunto de políticas económicas e industriales, así como las bases instrumentales que hicieran posible la posterior 
industrialización (Lucas, 2006). Las medidas propuestas para el sector manufacturero comprendían la organización de la producción nacional, el apoyo a los productores de materias primas, la protección de las industrias fabriles que existían y la promoción de otras nuevas (López Contreras, 1966).

En consecuencia, la visión de país de Eleazar López Contreras requería el intervencionismo de Estado, el cual se convirtió en la forma de accionar del gobierno para lograr objetivos económicos, sociales y políticos. Esta intervención se plasmó en las limitaciones formales que se introdujeron en el nuevo marco institucional ${ }^{8}$, que de acuerdo a lo señalado por North (1984, p. 39), especificó las "reglas fundamentales de competencia y cooperación para proporcionar una estructura de derechos de propiedad". También redujo la incertidumbre que generó el ascenso del gobierno de López Contreras. Este se distanció de la forma de hacer política de su antecesor el General Juan Vicente Gómez (1908-1935), quien en ocasiones desconocía la norma, en función de perpetuarse en el poder y de satisfacer sus intereses y la de sus familiares y amigos.

Las limitaciones formales e informales fueron esbozadas en la exposición de las líneas generales del Programa de Febrero9. De esta manera, se impulsó una importante emisión de decretos que permitió la implantación de los objetivos enunciados. El primero de ellos fue el Decreto del 25 de febrero de 1936, mediante el cual se creó el Ministerio de Agricultura y Cría, y que trató "la importancia del desarrollo racional de nuestra Agricultura y Cría", así como "la realización metódica de los planes del Ejecutivo Federal" (López Contreras, 1936, p. 49). Se puede señalar que en este decreto se introdujo la primera noción precursora sobre planificación en Venezuela.

Posteriormente, se realizó una reforma de la constitución, el 20 de julio de 1936, la cual estableció, entro otros aspectos, en el artículo 32, numerales 8, 9 y 10, cambios fundamentales en lo social, político y económico, al dejar establecidos los siguientes aspectos: La garantía y promoción a la libre iniciativa privada; la prohibición de monopolios; los privilegios temporales relativos a la propiedad intelectual; la justa protección al trabajador -mediante la legislación del trabajo; la creación del Consejo de Economía y la facultad del Poder Federal para reservarse el ejercicio de determinadas industrias para

8 De acuerdo con lo señalado por North (2007, pp. 86-87), "la estructura institucional formal de una sociedad está compuesta del marco constitucional concebido ampliamente, esto es, la estructura que define el modo como se pretende jugar el juego político y económico [...]. El modo concreto de jugar el juego es una consecuencia de la estructura formal, las restricciones institucionales informales y las características para garantizar el cumplimiento de las reglas". Asimismo, apuntó North (1995, p. 13), "las instituciones son las reglas del juego en una sociedad o, más formalmente, son las limitaciones ideadas por el hombre que dan forma a la interacción humana. Por consiguiente, estructuran incentivos en el intercambio humano, sea político, social o económico. El cambio institucional conforma el modo en que las sociedades evolucionan a lo largo del tiempo, por lo cual es la clave para entender el cambio histórico".

9 Un resumen amplio del Programa de Febrero se encuentra en "Programa de Febrero de López Contreras (1936): Importancia histórica", de Rondón de Medina y Rondón de Delgado (2012). 
asegurar los servicios públicos y la defensa y crédito de la Nación (Estados Unidos de Venezuela, 1936).

Este artículo de la constitución, así como los relativos al fomento de la actividad industrial, la protección y promoción del desarrollo agrícola, fueron consolidándose durante los años que siguieron. El 14 de octubre de 1937 presentó el Asesor Técnico del Ministerio de Agricultura y Cría, R. Alamo Ibarra (1937), un Plan de Orientación de Actividades del Ministerio, el cual expresó:

Como ejemplo de las campañas a desarrollar a favor de la producción agrícola y ganadera del país, se acompaña el esquema correspondiente a nuestro principal cultivo; el café. Este esquema pone de manifiesto los diferentes aspectos en que necesariamente debe ser considerada la Producción, la Circulación y el Consumo de dicho fruto para que pueda formularse luego un plan racional y armónico de actividades que resulte verdaderamente beneficioso para la Nación. De su examen podrá apreciarse fácilmente el positivo valor de los datos que habrá de proporcionar a tal respecto el Reconocimiento General de que se trata. Además, aparece claramente, la necesidad de cooperación de la campaña en referencia (medidas aduaneras, sanitarias, vías de comunicación, etc.) (p. 109).

Nótese cómo el asesor mencionado ya se refería a un plan racional beneficioso a la nación, por supuesto refiriéndose particularmente al café, rubro agrícola que ocupaba un lugar importante en la economía del país y que requería revitalizarse para retomar el lugar que había ocupado en el comercio internacional desde mediados del siglo XIX. Además, el plan de Alamo Ibarra revela la conciencia que existía en los dirigentes de la época sobre la racionalización en la toma de decisiones públicas, es decir, en el uso de la planificación como método para el logro de objetivos.

El desarrollo de la política industrial a partir del Programa de Febrero comenzó con la creación el $1^{\circ}$ de agosto de 1936 de la Dirección de Industria y Comercio, en el seno del Ministerio de Fomento. Ese mismo año se certificó la promoción del Banco Industrial y su constitución legal, con la cual se inició un proceso de crédito industrial. El 2 de marzo de 1937, por decreto del Ejecutivo Federal, se destinaron 5.000.000,oo de bolívares para garantizar el $50 \%$ de créditos industriales concedidos por institutos bancarios que permitió el otorgamiento de estos créditos. En consecuencia, se organizó un sistema de crédito industrial amplio y generoso con la colaboración del capital privado y del Estado que permitió el fomento de la producción (López Contreras, 1941, pp. 74-75).

En el año 1938 se cumplió y desarrolló la política de industria y comercio, cuando por iniciativa del gobierno de López Contreras quedó constituida la Cámara de Industriales y se especializó en "el esfuerzo protector en asegurar el futuro desarrollo de la principal industria nacional: la de manufacturas textiles". Este subsector industrial fue receptor de créditos por una cantidad de 800.000,oo bolívares, discriminados en 200.000,oo bolívares para la creación de la Compañía Fibro-textil del estado Lara y 600.000,oo bolívares para telares de Caracas y Valencia. Para octubre de este mismo año se estableció el 
contingente a la importación de los artículos textiles producidos por empresas venezolanas (López Contreras, 1941, p. 75).

Visto lo expuesto hasta este punto en lo relativo a las primeras acciones del Estado venezolano para introducir un cambio en el devenir de los acontecimientos, el camino se reforzó a partir de 1939 con el llamado Plan Trienal. En este plan se fortalecieron las medidas para el estímulo a la industria nacional que pasó a ocupar un rol más preponderante en la política de modernización del país. El sector industrial estaba representado fundamentalmente por empresas en los ramos de alimentos, química, vestido, cartón y papel, madera, vidrio, hierro, textiles, licorerías y destilerías. En este sentido, Egaña (1944) destacó el importante estímulo y fomento industrial "en virtud de la intervención protectora del gobierno en forma de cooperación técnica, mediante la concesión de créditos de largo plazo y una política de exoneraciones de derechos de importación sobre maquinarias y materias primas y de fuerte gravamen para el producto extranjero, lo cual reservó el mercado nacional al producto de la industrialización" (p. 143). Es decir, para 1939 el Plan Trienal prosiguió y amplió la racionalización del sistema de protección industrial, y diseñó un conjunto de medidas adicionales a las de crédito industrial.

\section{El Plan Trienal}

El Plan Trienal de 1939-1941 fue presentado por López Contreras al Congreso el 7 de mayo de 1938 como el plan administrativo "para dar así cumplimiento más efectivo al Programa de Febrero de 1936" (López Contreras, 1938, p. 243). En su presentación, señaló que para la elaboración del instrumento tomó en consideración los recursos del Estado, las posibilidades de crédito público y la participación de capitales privados, tanto venezolanos como extranjeros, pero dando preferencia a los primeros. Los objetivos fundamentales del plan eran lograr mayor producción y rendimiento de la economía nacional, abaratar el costo de la vida y sanear, educar y poblar. De esta manera, las previsiones del Plan Trienal persiguieron objetivos materiales concretos a diferencia del Programa de Febrero.

En lo relativo a "fomento y apoyo a la producción", se consideró primeramente el sector agrícola. Para ello se programó el mejoramiento de la producción agropecuaria, el mayor rendimiento de los cultivos, la incorporación de nuevos rubros agrícolas, el aumento de la producción del trigo, el arroz y otros cereales, con el fin de evitar su importación, la promoción de las exportaciones de bananos y tabaco y la eliminación del contrabando. Además, con el fin de aumentar la provisión de los productos agrícolas, se prescribió la realización de inventarios de las distintas riquezas, la protección sanitaria y vegetal, el montaje de estaciones experimentales de agricultura, la zootécnica y los campos ganaderos, la instalación de granjas agrícolas de demostración, la instauración de centros de enseñanza, de adiestramiento agropecuario y de centros de investigaciones 
veterinarias, el otorgamiento de servicios de higiene y sanidad animal, el fomento de la pesca y la piscicultura, el levantamiento de catastro de las tierras baldías, la recuperación de tierras que estaban en manos de particulares contentivas de hoyas hidráulicas, la reforestación de los bosques y la política de regadío. Finalmente, en esta materia se contemplaron subsidios como complemento especial de protección a la actividad agrícola nacional.

Igualmente el plan contenía indicaciones para la promoción industrial. A tal fin se precisó que las industrias nacionales recibirían decidida protección del Estado mediante préstamos a los industriales, la contratación de expertos para el ramo, y la ayuda para obtener maquinarias a quienes poseían pequeñas industrias y que procesaran materias primas nacionales. Esta ayuda se materializaría a través de la compra por parte del Estado de las máquinas requeridas, las cuales posteriormente serían vendidas por este en "condiciones módicas" a los industriales. La observancia de las medidas mencionadas llevó a Rivas Aguilar (1989) a considerar que el Plan Trienal fue la primera oportunidad en la que se expuso de "forma ordenada la exigencia de industrializar el país con el apoyo decidido del Estado” (p. 395). Además, destacó el papel de primer orden del Ministro Manuel Egaña, a quien le atribuyó el proyecto de industrialización del país.

En lo relativo a la industria petrolera, se estimó en el plan perfeccionar los instrumentos técnicos y legales que garantizarían la recaudación y el aumento de la renta, y se proyectó la instalación de una planta de refinería nacional de hidrocarburos. En materia de minería, se previó su desarrollo mediante el estudio de su potencial, la adquisición de equipos de exploración de suelos y subsuelos y acciones para la explotación, especialmente dirigida a Guayana, con participación compartida entre privados y el Estado; y, con respecto a la actividad turística, se programó el desarrollo de infraestructura hotelera y recreacional.

Finalmente, con efectos en la producción general se proyectó la construcción de vías públicas que facilitaran el comercio, el impulso de la red telefónica, radial, telegráfica y eléctrica en algunas ciudades vitales para la producción nacional, el mejoramiento de Puerto Cabello, el desarrollo de la navegación marítima y fluvial. También se consideró establecer acuerdos, tratados y convenios con distintas naciones para favorecer el impulso de la industria nacional mediante el comercio internacional, y se dispuso el fortalecimiento de la reglamentación de las relaciones capital-trabajo, con atención tanto al obrero urbano como al rural.

En relación con las políticas para "disminuir el costo de la vida", se estimaron medidas como la reducción del costo de los transportes y la revisión de "los costos adquisitivos para poner cese a las ganancias desmedidas de los intermediarios" (p. 256), así como la disminución de los derechos que gravaban los artículos de primera 
necesidad mediante el Decreto de 9 de septiembre de 1939 que estableció juntas reguladoras de precios en toda la república (López Contreras, 1941, p. 76). De otro lado, se contempló una revisión de la Ley de Arancel, en la cual se previó sustituir el sistema tarifario único por el de tarifas dobles que permitiera negociar útilmente con las demás naciones en materias de intercambio" (López Contreras, 1938).

Ahora bien, lograr el objetivo "sanear" implicó, por una parte, la higienización del hombre a través de la educación sanitaria y la profilaxia de endemias y epidemias, la asistencia médica, la construcción de hospitales y centros de asistencia social, la promoción de las instituciones protectoras de la madre y del niño, la provisión de sueros, vacunas y demás requeridos por las campañas sanitarias. Por la otra, la higienización del medio en que vivían los venezolanos supuso la provisión de aguas, el saneamiento del suelo, el abastecimiento el agua potable, la ejecución de cloacas y la eliminación de desperdicios. También significó atender la carrera sanitaria en sus distintos niveles formando médicos en distintas áreas, así como enfermeros, inspectores y laboratoristas, y la ejecución de una política de vivienda para los obreros y para la clase media. De igual forma reclamó el incremento de las comunicaciones postales, aéreas, telegráficas y telefónicas y mejoras de los medios de defensa nacional y de la policía interna; asegurar la paz era, para el gobierno, la condición esencial del progreso colectivo (López Contreras, 1938).

La otra tarea social que se propuso el Plan Trienal fue la de "educar" mediante la incorporación del mayor número de niños a la escuela primaria, el aumento del número de escuelas en todos los lugares del territorio nacional, la dotación con materiales didácticos y la formación de los docentes. También precisaba abocarse a la instrucción de los adultos y de los obreros, e incrementar el presupuesto de las universidades, a las que se les requirió la creación de facultades de ciencias económicas y sociales.

En relación con las llamadas escuelas especiales que incluían formación en áreas de administración, comercio, artes y oficios se fijaron mejoras, aunque el plan no especificó cuáles serían. Asimismo se declaró la necesidad de atender a las instituciones de carácter artístico y cultural, deportivas y a los boy-scouts.

Por último, el plan propuso "poblar". Partió de la consideración de que "la riqueza de un país reside más en el número y en la calidad de los hombres que en la abundancia de los elementos materiales de su suelo. Allí donde faltan hombres no hay riqueza posible y Venezuela no será jamás un gran país sin un paralelismo armonioso entre su potencialidad económica y el factor humano" (López Contreras, 1938, p. 251). Se previó entonces la creación del Instituto de Inmigración y Colonización que se encargaría de idear un sistema que facilitara a los inmigrantes mejores condiciones e incentivara sobre todo la incorporación a las tareas agrícolas. 
En función de lograr las metas descritas las asignaciones presupuestarias se plasmaron en el plan. Así, por ejemplo, se señaló que se construirían alrededor de 100 acueductos y 16 redes de cloacas, que se levantaría un sanatorio para la lucha antituberculosa en Caracas para 300 plazas, y otro en Mérida, de 120 puestos; y en cada uno de los cinco grandes hospitales que se construirían en Maracay, Barquisimeto, San Cristóbal, Cumaná y Ciudad Bolívar, se destinarían pabellones adecuados para esa campaña. Con el mismo fin se instalarían dispensarios especiales en San Felipe, Mérida, Coro, Barcelona y Carúpano.

En materia educativa se construirían 300 escuelas rurales y en cuanto al incentivo agrícola se instalarían 23 granjas agrícolas de demostración en diferentes puntos del territorio; se instituirían la Escuela Práctica de Agricultura de Maracay y el Centro de Demostración anexo; las escuelas de mayordomos para haciendas de café, en los estados Carabobo, Mérida, Táchira y Monagas, y las escuelas de mayordomos para haciendas de cacao. En esta misma línea se apoyarían los cultivos de bananos, tabaco, trigo, arroz y de otros cereales por medio del Banco Agrícola y Pecuario.

En el marco de la racionalización del Plan Trienal, se adjudicó una responsabilidad fundamental al Ministerio de Obras Públicas, que se encargó de ejecutar todas las obras de infraestructura requerida por los distintos ministerios. Para ello, fue necesario establecer criterios de jerarquización de prioridades y su respectiva estimación y asignación presupuestaria con base en criterios de eficiencia, y se distinguieron las obras de corto, mediano y largo plazo.

Es importante destacar que el plan culminaba con una lista detallada de las obras públicas previstas para el trienio, con su respectivo costo, indicando las obras que se financiarían con ingresos ordinarios y las que se ejecutarían en el largo plazo con recursos provenientes de financiamiento público. Un resumen de los rubros presupuestados se presenta en el Cuadro 1.

En términos presupuestarios la inversión realizada durante el periodo de 1936 a 1940 alcanzó la cantidad de 344.512.438,oo bolívares, que representaron el 22,1 \%, del total de ingresos obtenidos por el ejecutivo que fue 1.553.742.512,46 de bolívares. En contraste, en el lapso que va de 1870 a 1935, el gobierno nacional invirtió en obras públicas 668.521.669,oo bolívares, y recaudó ingresos por la cantidad de 4.651.763.855,05 bolívares, lo que equivale a un 14,3% de los ingresos públicos (López Contreras, 1941). Es decir que, en términos relativos, en solo cinco años se invirtió casi el doble en términos proporcionales de lo ejecutado en sesenta y cinco años, evidenciando la acción del Estado en el desarrollo de la infraestructura básica y de apoyo a la producción del país como aspecto preliminar de una política de crecimiento sostenido. 

en el Plan Trienal de López Contreras (1939-1941)

\section{Obras previstas a ejecutar con recursos ordinarios}

Monto en Bs

\section{Cloacas}

Defensa de Poblaciones

Obras de irrigación

Edificios de aislamiento, hospitalización, sanidad y asistencia social

Edificios para servicios del Ministerio de Relaciones Interiores

Edificios para servicios del Ministerio de Relaciones Exteriores

Edificios para servicios del Ministerio de Hacienda

Edificios para servicios del Ministerio de Guerra y Marina

Dependencias del Ministerio de Obras Públicas

Edificios para servicios del Ministerio de Educación Nacional

Edificios para servicios del Ministerio de Agricultura y Cría

Edificios para servicios del Ministerio del Trabajo y Comunicaciones

Edificios para otros servicios

Obras de embellecimiento en Caracas

Vías de comunicación terrestres

Caminos de herradura

Construcciones en las carreteras ya en actividad

Obras complementarias

Conservación y mejoras de carreteras

Estudios de carreteras (contratados)

Obras relativas a la Navegación Marítima y Fluvial

Obras relativas a la Navegación Aérea

Total

\section{Obras que se ejecutaran dentro del Plan Trienal con financiamiento a Largo Plazo (20 años)}

Ferrocarriles

Puertos

Salinas de Araya

Acueductos

Hoteles y Balnearios (Turismo)

Sub Total

Total general del plan trienal
16.299.630

5.400 .000

3.000 .000

16.065 .000

10.040 .200

974.000

700.000

10.111 .400

454.000

17.780 .000

2.996 .000

860.000

2.526 .000

4.750 .000

33.123 .770

2.844 .100

4.201 .500

8.840 .325

10.064.397

220.400

5.400 .000

3.500 .000

160.150 .722
61.000 .000

101.500 .000

6.000 .000

30.000 .000

5.865 .000

204.365.000

364.515.722

Fuente: López Contreras, 1938. 
La descripción de obras de infraestructura previstas en el Plan Trienal revela la importancia de la subestructura, de los medios, de las instalaciones y de los servicios, sobre los que se sustentaba la visión de progreso del gobierno de Eleazar López Contreras que, adicionalmente, constituían un aspecto esencial para la transformación del país, evidenciando lo referido por Tinberger (1959): "Para que un país tenga estabilidad y proporcione una base para la actividad y el desarrollo económico es preciso hacer una serie de inversiones fundamentales. Debe haber un mínimo de alojamiento y de transportes. El abastecimiento de energía y agua debe existir en forma adecuada; es posible que sea preciso abrir nuevas tierras o regarlas, etc." (p. 10). De esta forma, con estas inversiones oficiales básicas del plan, se procuraba brindar una plataforma para el desarrollo general del país.

Con respecto a los objetivos sociales y económicos previstos en el plan, igualmente estos tuvieron una importante asignación presupuestaria para desarrollar las medidas descritas, algunas de las cuales condujeron a la elaboración de planes específicos para atender casos particulares, tal como el Plan de Urbanismo de Caracas (1939). Cabe destacar que Luis Lander y María Josefina Rangel (1970) apuntaron que este plan, presentado por el gobernador de dicha entidad, fue el primer intento de planificación del país, cuestión que aquí no se comparte en virtud de considerar que dicha categoría corresponde a mecanismos de acción pública que tienen un impacto general en los distintos ámbitos y dimensiones de un país, como los descritos en el Programa de Febrero, extendidos en el Plan Trienal. En cambio, un plan de urbanismo es un instrumento de organización físico espacial que comprende, esencialmente, la ubicación de las actividades productivas y residenciales.

Indiscutiblemente, las obras dieron resultados. Por ejemplo, en el área educativa las diversas medidas que pueden verse en el Cuadro 2, produjeron una disminución considerable del analfabetismo. Así, en el año 1936 el 36,3 \% de la población sabía leer, y para 1940 alcanzaba el $48 \%$. Por consiguiente, se redujo el porcentaje de analfabetos de 63,7 \% a 52 \% aproximadamente (López Contreras, 1941). Otra evidencia de los logros fue el aumento de la matrícula escolar promedio quinquenal, que para los años 1926 a 1935 alcanzó 550.000 inscritos, mientras que para el quinquenio 1936 a 1940 fue de 1.160 .000 inscritos. Todo ello requirió un incremento del gasto público que elevó el presupuesto promedio quinquenal entre los años 1926 a 1935 de 37.000 .000 bolívares a 110.000.000 en el gobierno de López Contreras.

Con respecto al área de salud (sanidad y asistencia social) los programas se destinaron a combatir la tuberculosis, el paludismo, las enfermedades venéreas, la buba, la fiebre amarilla, la anquilostomiasis, el cáncer y la lepra (López Contreras, 1941). Ello afectó positivamente el crecimiento de la población (ver Cuadro 2) y, por ende, la fuerza de trabajo. En este sentido, el Censo de 1936 arrojó que la población total para ese año era 
de 3.364.347 de habitantes, mientras que el Censo de 1941 registró que el número de habitantes fue de 3.850.781 (Egaña, 1944).

En cuanto a la promoción industrial resalta que, tal como prescribió el Plan Trienal, los recursos del Estado se pusieron al servicio de la promoción de la industria. Entre 1939 y 1940 la protección otorgada a la industria estuvo conformada por garantías ofrecidas a créditos industriales por 1.253.000,oo bolívares; préstamos directos y suscripciones en beneficio y promoción industrial por 14.225.000,oo; obligaciones contratadas por 5.985.000,oo; créditos directos a la pequeña y mediana industria por 2.040.680,oo; créditos a favor de la producción por 9.742.531,82 que representaron el $3 \%$ del gasto público. Las industrias favorecidas estaban representadas por: Alimento, química, vestido, cartón y papel, madera, vidrio y cerámica, hierro, textiles, licorerías y destilerías, entre otros (López Contreras, 1941, p. 104).

Cuadro 2. Comparación de las principales obras realizadas en distintas áreas sociales de 1935 y 1941

\begin{tabular}{|l|c|c|}
\hline Educación & $\mathbf{1 9 3 5}$ & $\mathbf{1 9 4 1}$ \\
\hline Escuelas Primarias Federales Existentes & 1.430 & 2.431 \\
\hline Maestros existentes en las Escuelas Federales & 1.915 & 3.772 \\
\hline Escuelas Normales existentes & 3 & 19 \\
\hline Número de alumnos inscritos en las Escuelas Normales & 161 & 1.138 \\
\hline Escuelas Primarias existentes & 2.161 & 5.647 \\
\hline Sanidad y Asistencia Social & 29,6 & 39,8 \\
\hline Nacimientos Coeficiente por 1.000 habitantes para toda la Republica & 22,9 & 19,3 \\
\hline Mortalidad general Coeficiente por 1.000 habitantes para toda la Republica & 7 & 20,3 \\
\hline Crecimiento vegetativo Coeficiente por 1.000 habitantes para toda la Republica & 213 \\
\hline Hospitales y asilos & 51 & 14.304 \\
\hline Número de camas en los hospitales y asilos & 3.653 \\
\hline Instituciones para la lucha contra la tuberculosis & 2 & 25 \\
\hline
\end{tabular}

Fuente: López Contreras (1941)

En última instancia, debe señalarse que el Plan Trienal trascendió su exposición y efectivamente tuvo alcances concretos. Sin ser el propósito de este estudio evaluarlos, debe señalarse que las obras programadas en la gestión de López Contreras fueron ejecutadas en su mayoría y las que no se dejaron iniciadas. En consecuencia, el General López Contreras logró la construcción material del país y, si bien no alcanzó a atender todos los requerimientos de los distintos sectores, se puede estar de acuerdo con Chiossone (1989) cuando expresa: 
La construcción de un país que sea habitable, que vaya respondiendo día a día a las exigencias del progreso y las necesidades colectivas, no puede ser obra de un solo Gobierno. Pero sí puede ser labor de uno solo la iniciación de un proceso constructivo, al suplir las necesidades del presente en que actúa, y dejar sentada, en lo material, la planificación inteligente de lo que el país necesita (p. 112).

\section{Conclusiones}

Las percepciones subjetivas de los intelectuales y los políticos sobre la situación de América Latina y la manera de resolverla, marcaron el tipo de planificación que adoptó la región y produjeron un nuevo marco institucional. De esta forma, múltiples reflexiones y debates llevaron a concluir que dadas las características estructurales de Latinoamérica, no podían seguir las medidas de corto y mediano plazo que se habían aplicado en los países avanzados. Se requería una programación general con una visión de largo plazo que impactara los distintos órdenes de la vida social.

El conjunto de las medidas sistematizadas para producir el cambio institucional fueron expuestas por la CEPAL, y estas fueron adoptadas por los países de la región que establecieron un conjunto de limitaciones formales e informales, dadas las recomendaciones formuladas por este organismo. Dadas estas circunstancias, el proceso de implantación de la planificación en América Latina fue conformándose a partir del cambio en el sistema de creencias de los líderes encargados de procurar las transformaciones necesarias que impulsaron los mecanismos de Estado para propiciar la industrialización. Este proceso puede definirse, siguiendo los constructos de North (1993), como un proceso de cambios institucionales fundamentales que resultarían en un desempeño económico eficiente, en virtud de recursos humanos en cantidad y calidad suficientes, acumulación de conocimientos y mejora del progreso técnico, aumento de la productividad y aumento de la participación en el comercio internacional en condiciones beneficiosas para la región. Es decir, en un crecimiento económico sostenido.

En el caso venezolano, a partir de 1936 con el ascenso al poder de López Contreras, se realizaron los primeros esfuerzos para emplear métodos racionales de conducción de las fuerzas productivas. Por consiguiente, aun cuando el Sistema Nacional de Planificación, impulsado por la CEPAL se creó en 1958, desde 1936 se marcó la vía para atender racionalmente la toma de decisiones públicas, con lo que las acciones instrumentadas en el gobierno lopecista tienen carácter precursor en esta materia.

En cuanto a los emprendedores políticos que tuvieron un rol protagónico y pionero en la tarea de cambiar el sistema de creencias en torno a la intervención del Estado, destacan Alberto Adriani y Manuel Egaña, quienes creían en la pertinencia de una acción estatal racional, metódica, armónica y general para transformar la realidad del país. Sus ideas fueron consideradas en el Programa de Febrero, dado que al formar parte de la comisión gubernamental encargada de elaborarlo, pudieron exponer su visión. Es decir, 
en Venezuela entre 1936 y 1941 se acometieron acciones similares a las emprendidas por los países adelantados de la región (Argentina, México, Brasil y, en menor medida, Colombia) en materia de bienestar social, estímulo a la producción agrícola e industrial y a la modernización de las instituciones.

El examen de la gestión del Presidente López Contreras permite concluir que desde la perspectiva de la planificación, el esfuerzo político administrativo expuesto en su Programa de Febrero y en su Plan Trienal, introdujeron los elementos iniciales para la construcción de un sistema nacional de planificación de Venezuela. El primer paso de este proceso fue el diagnóstico sobre la situación del país y las medidas que debían emprenderse para lograr su transformación, plasmado en el Programa de Febrero. Consecuentemente, el segundo paso del proceso fue la asignación presupuestaria para la ejecución de obras, expuesta en el Plan Trienal.

Por consiguiente, el ascenso al poder del General Eleazar López Contreras en 1936 inauguró una nueva etapa en la historia de Venezuela. Su gestión encabezó la ola de cambios que el país requería para generar la transformación social, política y económica que exigía su modernización. El programa impulsó un nuevo marco institucional que, de acuerdo a lo señalado por North (2007, p. 86), implicó el establecimiento de un conjunto de limitaciones formales e informales, liderizadas por la constitución que definió el modo como se jugaría el juego político y económico.

Estas reformas implicaron mayor intervención del Estado en la vida social y económica del país, justificada en aspectos tanto externos como internos. Entre los primeros se encontraban la Crisis del 29, el inicio de la Segunda Guerra Mundial y el cambio de visión en torno a la actuación del Estado, que pasó del liberalismo economismo al intervencionismo. Entre los segundos, la urgencia de mejorar las condiciones de vida de la población, la necesidad de proteccionismo e impulso a la agricultura y a la industria nacional, el desarrollo de la infraestructura básica del país se convirtieron en los aspectos fundamentales que llevaron a diseñar los instrumentos de acción de la política venezolana para garantizar la superación de la crisis nacional, caracterizada por el atraso.

De esta manera, el Programa de Febrero y el Plan Trienal expresaron la acción racional del Estado para conducir las fuerzas económicas de Venezuela y enrumbarla en el camino del progreso. Estos instrumentos de gestión establecieron un conjunto de medidas que de acuerdo a lo señalado por Tinberger (1958), procuraron el diseño de una "política de desarrollo" por cuanto i) crearon condiciones generales favorables al progreso; ii) propiciaron una conciencia de las potencialidades y ventajas del desarrollo; iii) realizaron importantes inversiones básicas, e iv) instrumentaron medidas para estimular la actividad privada. 
En cuanto a la creación de condiciones generales, las primeras acciones instrumentadas a partir del Programa de Febrero produjeron reformas en el marco institucional para asegurar condiciones de legalidad, seguridad y estabilidad. De esta manera en la Constitución de 1936 se establecieron aspectos como la seguridad física de las personas, el mantenimiento de estabilidad política, la promoción del establecimiento de organizaciones políticas y gremiales, lo relativo a aspectos económicos, el respeto a la propiedad privada, la prohibición de monopolios, la instauración de derechos laborales y el fomento de las relaciones entre capital y trabajo. Igualmente, es de resaltar la implantación de instrumentos de "política económica" como la política fiscal, la cambiaria, la arancelaria y la creación del Banco Central. Todo ello con el fin de lograr el bienestar de la población.

En lo relativo a la conciencia de las potencialidades y ventajas del desarrollo, conviene señalar que no se manejaba para la década del treinta el concepto de desarrollo, el cual se conformó teóricamente a partir de la década del cincuenta del siglo veinte. Sin embargo, en la visión del General López Contreras, el progreso y el desenvolvimiento económico eran objetivos claros, expresados reiteradamente por el presidente y que se convirtieron en los fines de las medidas y reformas instrumentadas. Además, se hizo uso de "las estadísticas, la investigación y la información" (Tinberger (1958, p. 9), como herramientas para la toma de decisiones, dado que durante el gobierno de López Contreras se realizaron esfuerzos por implantar el sistema de estadísticas centralizadas de la nación.

En este orden de ideas, se dictó la Ley de Estadística el 22 de agosto de 1938 y se contrató a un experto en la materia, José Vandellós, quien estuvo a cargo de dirigir y organizar tanto el Primer Censo Industrial Comercial como el Censo Agrícola y Pecuario, realizados en 1936. A partir de enero de 1939 se inició la estadística del movimiento demográfico en función de "boletas individuales" para los nacimientos, los matrimonios y las defunciones, y se comenzaron a elaborar índices económicos, tales como:

El de los precios al por mayor, de las subsistencias, el de cotización de acciones y valor oro del Bolívar, el de producción de petróleo y oro, el del valor de las exportaciones de café y cacao, valor de las hipotecas y cancelaciones, carga por ferrocarril e importación de autocamiones, ingresos y egresos del Tesoro, billetes en circulación y oro en los Bancos, cuentas corrientes del Activo y Avances, depósitos bancarios, producción de cemento, principales fuentes de ingresos del petróleo y los índices del movimiento demográfico (López Contreras, 1941, pp. 79-80).

Este esfuerzo estadístico le permitió al gobierno contar con la información necesaria para diseñar las políticas a seguir.

Con respecto a las inversiones básicas, con el Plan Trienal el gobierno de López Contreras ejecutó un destacado programa de inversiones básicas y de apoyo a la producción que no tenían precedentes en el país. La visión progresista del gobierno lo llevó a considerar 
tanto lo relativo a la infraestructura básica para la actividad económica, vías y medios de comunicación, puertos, aduanas, abastecimiento de energía y agua, como la necesidad de mejorar el regadío de tierras, la incorporación de nuevas tierras a la producción nacional, el resguardo de áreas especiales y la inversión en organismos para el apoyo técnico y la investigación en diferentes ramas económicas. Igualmente importantes fueron las inversiones para asegurar la superación de las adversas condiciones educativas y de salud de la población, en un esfuerzo por procurar ciudadanos sanos y educados.

Por último, en lo referente a las medidas para facilitar y estimular la actividad privada, el esfuerzo de inversión realizado por López Contreras, a partir de 1939, se orientó a la promoción de la actividad privada mediante créditos, subsidios y apoyo técnico a productores agrícolas e industriales, así como el desarrollo de la industria petrolera y minera del país, en un dedicado esfuerzo por generar condiciones deseables para la creación y desarrollo de empresas, la generación de empleo y el aumento de la producción nacional.

Conviene señalar que todas estas medidas estuvieron enmarcadas dentro de una acción intervencionista que se hizo explícita no solo en el discurso de su principal dirigente, sino en el diseño e implantación de las acciones del Estado. La guía de implantación quedó plasmada en los dos documentos que se han venido analizando. Paradójicamente, desde la década del veinte se contaron con recursos provenientes del sector petrolero que no fueron dedicados por el gobierno gomecista para generar las transformaciones fundamentales, sino para garantizar su permanencia en el poder.

De esta forma, el gobierno de López Contreras sustituyó un gobierno de corte dictatorial cuyos objetivos estaban distanciados de las acciones conducentes a lograr el bienestar general. Por ello tiene el mérito de emprender una gestión que produjo un cambio institucional que respondió a una concepción racional, pensada desde el Estado y que lo llevó a ejecutarla con un sentido de responsabilidad histórica sin precedentes en la vida nacional. Obviamente, este cambio institucional no alcanzó a incursionar en los terrenos políticos y democráticos que llegarían en el periodo 1945-1948. 


\section{Referencias}

Adelman, I. (1961). Teorías del desarrollo. México: Fondo de Cultura Económica.

Adriani, A. (1984). "La primera etapa de una política económica", pp. 101-106. En Labor Venezolanista (1984). Caracas: Academia Nacional de Ciencias EconómicasConsejo de Profesores Universitarios de la Universidad Central de Venezuela.

Adriani, A. (1984). Labor venezolanista. Caracas: Academia Nacional de Ciencias Económicas-Consejo de Profesores Universitarios de la Universidad Central de Venezuela.

Adriani, A. (1984). "La valorización del café y nuestra economía nacional”, pp. 181189. En Labor venezolanista. Caracas: Academia Nacional de Ciencias EconómicasConsejo de Profesores Universitarios de la Universidad Central de Venezuela.

Adriani, A. (1922). "La nueva Alemania y Walter Rathenau", pp. 81-92. En Labor Venezolanista (1984). Caracas: Academia Nacional de Ciencias Económicas-Consejo de Profesores Universitarios de la Universidad Central de Venezuela.

Alamo Ibarra, R. (1937). "Organización del plan de orientación de actividades del Ministerio de Agricultura y Cría”, pp. 109-110. En Gobierno y época del presidente Eleazar López Contreras: Decretos y memorias del ejecutivo nacional (1935-1941). Pensamiento Político Venezolano del Siglo XX (1985). VII, II, 18. Caracas: Congreso de la República, Ediciones Conmemorativas del Bicentenario del Natalicio del Libertador Simón Bolívar.

Alianza para el Progreso (s. f.). Documentos básicos. Punta del Este, Uruguay.

Bénard, J. (1965). "Los métodos de la planeación francesa y su adaptación a los países en vías de desarrollo". Programación del desarrollo económico. México: Fondo de Cultura Económica.

Borbély, S. (1991). Un concepto económico y social latinoamericano. El Modelo Prebisch-CEPAL. Caracas: Universidad Simón Bolívar, Instituto de Altos Estudios de América Latina, Fundación Bicentenario de Simón Bolívar.

Chiossone, T. (1989). El decenio democrático inconcluso. Eleazar López Contreras e Isaías Medina Angarita. Caracas: Editorial Ex Libris.

Congreso de la República (1985). Gobierno y época del presidente Eleazar López Contreras: Decretos y memorias del ejecutivo nacional (1935-1941). Ediciones Conmemorativas del Bicentenario del Natalicio del Libertador Simón Bolívar. Caracas. 
ISSN: 0124-3551 / Año 15, No 23 / julio-diciembre / pp. 49-76

Crazut, Rafael J. (1989). Manuel R. Egaña. Obra y pensamiento. Caracas: Academia Nacional de Ciencias Económicas.

Egaña, M. (1944). "Venezuela en cifras", Revista de Fomento, (1944), pp. 118-139.

Estados Unidos de Venezuela (1936). Constitución de 1936. Biblioteca Virtual Miguel de Cervantes, fecha de consulta 12 de enero de 2013. Recuperado de: http:/ / bib. cervantesvirtual.com/servlet/SirveObras/0148274156783594 6320035/p0000001. htm\#I_1_

Junta de Gobierno de la República de Venezuela (1958). “Decreto 492 de 1958” (30 de diciembre de 1958).

Lander, L. y Rangel, María J.(1970). La planificación en Venezuela. Caracas: Sociedad venezolana de planificación.

Lira, L. (2006). Revalorización de la planificación del desarrollo. Serie Gestión Pública, 59. Santiago de Chile: Instituto Latinoamericano y del Caribe de Planificación Económica y Social (ILPES)-Naciones Unidas.

López Contreras, E. (1966). "Programa de Febrero". En Gobierno y administración 1936 1941. Caracas: Editorial Arte.

López Contreras, Eleazar (1941). Mensaje que el ciudadano General E. López Contreras Presidente de los Estados Unidos de Venezuela, presenta al congreso nacional en sus sesiones ordinarias de 1941. Caracas: Litografía del Comercio.

López Contreras, E. (1938). "Intervención del Presidente de la República, General Eleazar López Contreras, al exponer ante el Congreso Nacional su Plan Trienal Político-administrativo. Sesión del 7 de mayo de 1938”. Pensamiento político venezolano del Siglo XX. Documentos para su estudio. Gobierno y época del presidente Eleazar López Contreras. Mensajes y memorias 1935-1941. 17. Caracas: Congreso de la República, Ediciones conmemorativas del Bicentenario del Natalicio del Libertador Simón Bolívar, 1985.

López Contreras, E. (1936). "Creación del Ministerio de Agricultura y Cría”. Caracas, 25 de febrero de 1936, pp. 49-52. En Gobierno y época del presidente Eleazar López Contreras: Decretos y memorias del ejecutivo nacional (1935-1941). Pensamiento Político Venezolano del Siglo XX (1985). VII, II, 18. Caracas: Congreso de la República, Ediciones Conmemorativas del Bicentenario del Natalicio del Libertador Simón Bolívar.

Lucas, G. (2006). Industrialización contemporánea en Venezuela: Política industrial del Estado venezolano 1936-2000. Caracas: Universidad Católica Andrés Bello. 
Maldonado-Veloza, F. (2012). "Los conflictos alrededor del 'cambio institucional' petrolero venezolano entre 1936 y 1943 [Primera parte]". Revista CIFE: Lecturas de Economía Social (Universidad Santo Tomás), 14, 20 (enero-junio, 2012), pp. 27-54.

Maldonado-Veloza, F. (2012). "Los conflictos alrededor del 'cambio institucional' petrolero venezolano entre 1936 y 1943 [Segunda parte]." Revista CIFE: Lecturas de Economía Social (Universidad Santo Tomás), 14, 21 (julio-diciembre, 2012).

Matus, C. (1981). Estrategia y plan. México: Siglo Veintiuno Editores S.A.

Ministerio de Fomento (1940). Memoria y cuenta presentada ante el congreso de los Estados Unidos de Venezuela. Caracas: Cooperativa de Artes Gráficas.

Ministerio de Fomento (1939). Memoria y cuenta presentada ante el congreso de los Estados Unidos de Venezuela. Caracas: Cooperativa de Artes Gráficas.

North, Douglass, C. (2007). Para entender el proceso de cambio económico. Traducción de Horacio Pons. Edición económica de Juan Carlos Echeverry. Bogotá: Grupo Editorial Norma.

North, Douglass, G. (1993). Instituciones, cambio institucionaly desempeño económico. Traducción de Agustín Bárcena. México: Fondo de Cultura Económica, 1993, p. 190. [Edición original en inglés, 1990].

North, Douglass (1984). Estructura y cambio en la historia económica. Traducción de María Dolores Dionis y Fernando Fernández Méndez de Andés. Madrid: Alianza Editorial, 1984, p. 244. [Edición original en inglés, 1981].

Parra Luzardo, G. (2006). "Contribuciones a la integración de América Latina para emprender una tarea original”. Raúl Prebisch. Pensamiento renovador. Colección Científicos Sociales Latinoamericanos. Caracas: Banco Central de Venezuela.

Prebisch, R. (1983). "Cinco etapas de mi pensamiento sobre el desarrollo." El Trimestre Económico, 1, 198 (1983), pp. 1077-1096.

Rivas Aguilar, R. (1989). "Los orígenes del intervencionismo estatal en Venezuela (19361941)". Revista Venezolana de Ciencia Política. II, 3, diciembre, CEPSAL-ULA, Mérida.

Rivas Aguilar, R. y Maldonado-Veloza, F. (2010). "Dos políticas económicas y un mismo bolivarianismo: (1936-1945) vs. (1999-2009)". Tercer lugar del Concurso de Ensayo Libre "José Antonio Páez" sobre Historia Económica. Centro de Divulgación del Conocimiento Económico para la Libertad (CEDICE Libertad), Academia Nacional de Ciencias Económicas, Centro de Estudios Latinoamericano Arturo Uslar Pietri 
ISSN: 0124-3551 / Año 15, No 23 / julio-diciembre / pp. 49-76

(CELAUP), Facultad de Ciencias Económicas y Sociales de la Universidad Central de Venezuela, Caracas, 15 de diciembre del 2009.

Rondón de Medina, V. y Rondón de Delgado, Luz M. (2012). "El Programa de Febrero de López Contreras (1936): Importancia histórica”. Revista Venezolana de Ciencia Política. 42 (en prensa).

Szinetar Gabaldón, M. (1998). El proyecto de cambio social de Alberto Adriani 1914-1936). Caracas: Centros de Estudios del Desarrollo (CENDES), Universidad Central de Venzuela.

Tinbergen, J. (1959). La planeación del desarrollo. México: Fondo de Cultura Económica.

Todaro, M. (1988). El desarrollo económico del Tercer Mundo. Madrid: Alianza Editorial. 